\title{
Measurement of the electroweak WW production and production cross section of dijets associated with vector bosons.
}

\author{
Julia FISCHER* \\ on behalf of the ATLAS and CMS Collaborations \\ Bergische Universitaet Wuppertal \\ E-mail: fischerephysik.uni-wuppertal.de
}

\begin{abstract}
This article presents electroweak measurements from the ATLAS and the CMS experiment with the main focus on the pure electroweak $Z$ boson production in association with two jets. Besides the cross section measurement at $\sqrt{s}=8 \mathrm{TeV}$ with an integrated luminosity of $20.3 \mathrm{fb}^{-1}$ taken at the LHC in 2012, other interesting studies like gap fraction properties are discussed.

We also present the first evidence for same sign $W W$ scattering from the ATLAS experiment with the full dataset of $20.3 \mathrm{fb}^{-1}$ at $\sqrt{s}=8 \mathrm{TeV}$ and the search for exclusive two photon production of $W^{+} W^{-}$, performed by CMS with $5.05 \mathrm{fb}^{-1}$ at $\sqrt{s}=7 \mathrm{TeV}$.
\end{abstract}

XXII. International Workshop on Deep-Inelastic Scattering and Related Subjects 28 April - 2 May 2014

Warsaw, Poland

${ }^{*}$ Speaker. 


\section{Introduction}

The outstanding performance of the LHC in 2011/2012 and the large amount of data recorded by the ATLAS [1] and the CMS [2] experiment allows to observe even rare processes, like pure electroweak interactions of vector bosons and jets, which are sensitive to anomalous couplings of the vector bosons and other kinds of physics beyond the Standard Model. We present the pure electroweak $Z$ boson production in association with two jets from the ATLAS and the CMS experiment at $\sqrt{s}=8 \mathrm{TeV}$ and the first evidence for same sign $W W$ scattering from the ATLAS experiment with the full dataset of $20.3 \mathrm{fb}^{-1}$ at $\sqrt{s}=8 \mathrm{TeV}$ as well as the search for exclusive two photon production of $W^{+} W^{-}$performed by CMS with $5.05 \mathrm{fb}^{-1}$ at $\sqrt{s}=7 \mathrm{TeV}$

\section{Electroweak production of $Z$ bosons in association with two jets}

The dominant production process for a $Z$ boson at the LHC is via the Drell Yan process. Additional jets arise from strong interactions like gluon radiation or gluon splitting. A $Z$ boson in association with two jets can also be produced in pure electroweak processes via t-channel exchanges of a vector bosons. As these processes contain no strong interactions they are much rarer than the $Z$ boson +2 jets production including strong interactions. Examples of such processes are shown in figure 1.

The most interesting pure electroweak process is the feynman diagram shown on the left side of figure 1 , where the $Z$ boson is produced via Vector Boson Fusion (VBF) of two $W$ bosons. The two jets in the final state, also called the "tagging" jets, occur directly from the incoming quarks and show a characteristic kinematic. They are often scattered in the very forward and backward region of the detector. Since there is no color exchange between the incoming quarks, no hadronic activity is expected in
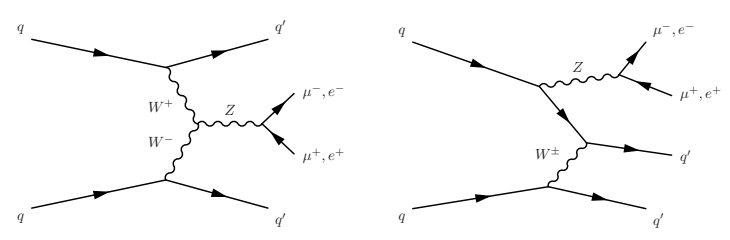

Figure 1: Feynman diagrams of pure electroweak $Z+$ two jets production: The left hand side shows the VBF process, where the $Z$ boson is produced via the fusion of two Vector Bosons. The right hand side shows a colorless $t$-channel exchange of a $W$ boson, where the $Z$ boson is radiated from one of the quark lines.

the central region of the detector. The large angle between the two jets leads to a large invariant mass of the dijets system, which allows to identify the VBF process. This process is similar to the Higgs production via Vector Boson Fusion and can therefore be used to understand the kinematics of the "tagging" jets, which is needed to study the Higgs boson properties, especially in models where the Higgs boson does not couple to leptons and the production via VBF become the dominant process. The right hand side of figure 1 shows the $\mathrm{t}$ channel excange of the $W$ boson, in this process the $Z$ boson is radiated from one of the quark lines. The processes in figure 1 have a large interference so they can not be calculated separately.

The largest challenge for measuring the pure electroweak process is to separate it from the large background of $Z$ boson with two quarks from strong interactions. In the following the different approaches for the cross section measurement from both experiments ATLAS and CMS and some highlights from studies on the hadronic interaction between the tagging jets are discussed. 


\subsection{Results from the CMS Experiment}

The CMS Experiment measured the cross section of the electroweak $Z+2$ jets in two different kinematic regions with different approaches. The first measurement is only performed in the $\mu \mu$ channel by fitting the signal in the Boosted Decision Tree (BDT) output distribution. The most important distributions to train the BDT, which separates the signal from the large background, are the jet variables. This is due to the different kinematics in the signal and the background processes.
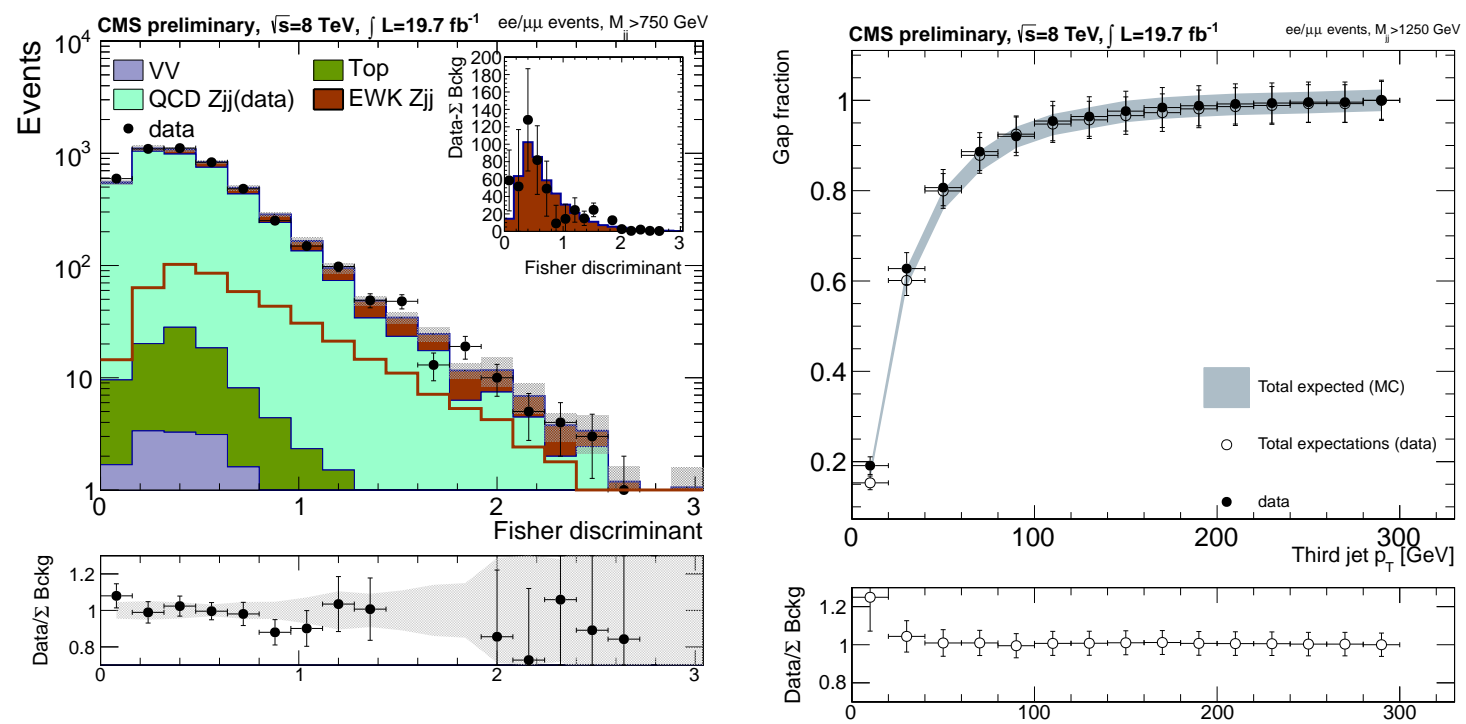

Figure 2: Fisher shape discriminator used for both lepton channels in the most sensitive region $\left(m_{j j}>\right.$ Figure 3: Gap fraction for the pt of the third jet. Com$750 \mathrm{GeV}$ ). The inset displays the result of a bin-by- pared to a data driven and a MC based background bin background subtraction prediction.

The second approach is done for high invariant dijet mass only $\left(m_{j j}>750 \mathrm{GeV}\right)$, where both lepton channels are taken into account. The jets kinematics are still the most important variables to determine the Fisher discriminant, which is shown in figure 2. The inset displays the result of a bin-by-bin background subtraction.

With the BDT the inclusive cross section is measured to $\sigma(\mathrm{EWK} Z+\mathrm{jj})=191 \pm 29_{\text {stat }} \pm 39_{\text {syst }} \mathrm{fb}$ and with the Fisher discriminant $\sigma(\mathrm{EWK} Z+\mathrm{jj})=303 \pm 29_{\text {stat }} \pm 57_{\text {syst }} \mathrm{fb}$. These two results can be combined to $\sigma(\mathrm{EWK} \mathrm{Z}+\mathrm{jj})=226 \pm 26_{\text {stat }} \pm 35_{\text {syst }} \mathrm{fb}$ which is in good agreement with the theoretical cross section at Next-to-Leading-Order of $239 \mathrm{fb}$.

In addition to the pure cross section determination the analysis also performed measurements of hadronic activity in a high purity region with $m_{j j}>1250 \mathrm{GeV}$. Figure 3 shows the gap fraction as a function of the $p_{\mathrm{T}}$, where the gap fraction is defined as the number of events with no jet above a given threshold. The data are compared to two different background predictions, a MC based and a data driven one and a good agreement between data and the sum of the backgrounds is found. More details of the CMS measurement can be found in [5] and [4].

\subsection{Results from the ATLAS Experiment}

Besides the cross section measurement of $Z+2$ jets in five fiduical regions with different sensitivity to the pure electroweak component, ATLAS provides also the unfolding of various VBF sen- 


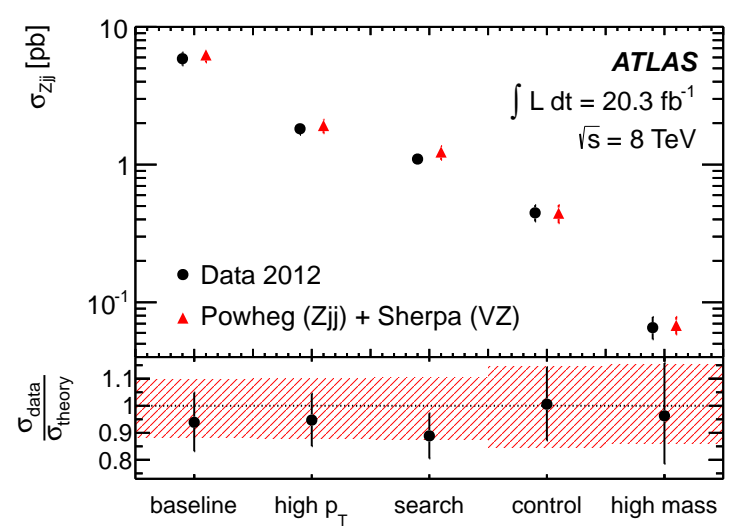

Figure 4: Cross section measurement in 5 fiducial regions: Good agreement of the measured cross section with the theory prediction from Powheg and Sherpa is found in all phase spaces.

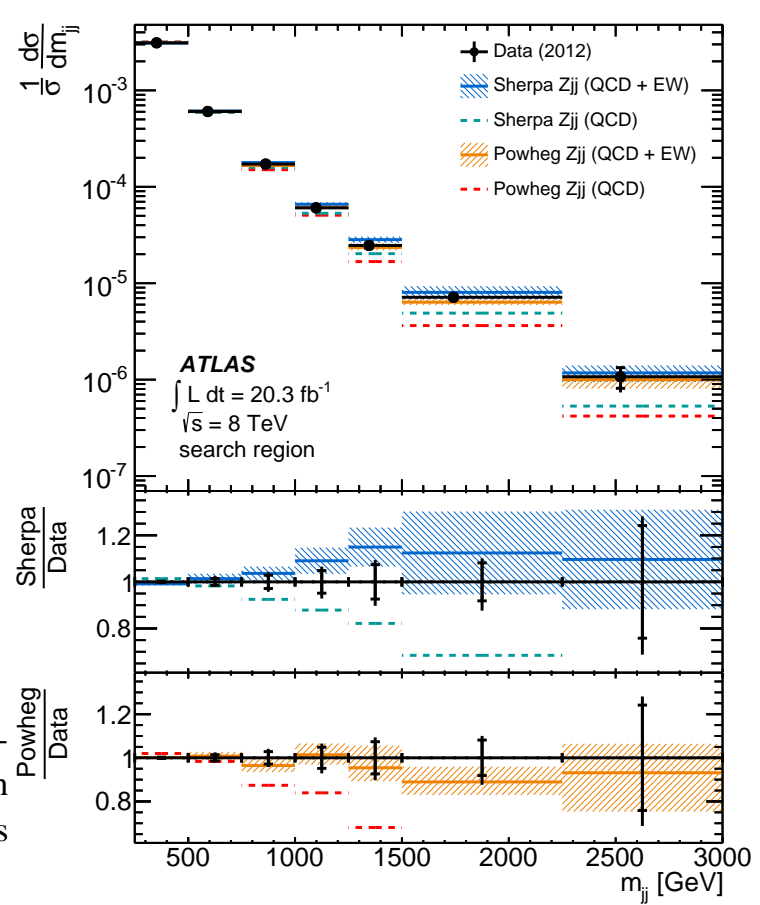

Figure 5: Unfolded $m_{j j}$ distribution compared to Powheg and Sherpa.

sitive distributions and limits on anomalous Triple Gauge Couplings. Figure 4 shows the results of the cross section measurement in the different kinematic regions. Apart from the baseline selection which requires only a nominal $Z+2$ jets selection with two isolated leptons and two jets, the "high $p_{\mathrm{T}}$ " region cuts at $p_{\mathrm{T}}\left(j_{1 / 2}\right)>85 / 75 \mathrm{GeV}$, while the "high mass" region cuts at $m_{j j}>1 \mathrm{TeV}$. The last two region are optimised to enhance the fraction of the pure electroweak component ("search region") and to suppress it ("control region"), by suppressing or requiring a third jet between the tagging jets. In the control region the background modelling is studied and could be improved by a reweighting procedure, which is then also applied in the signal region. The cross section in all fiducial regions is extracted by a fit to the invariant mass distribution of the dijet system. Figure 4 shows that in all phase spaces the measured cross section in data is in good agreement with theory prediction from Powheg and Sherpa.

Figure 5 shows the unfolded invariant dijet mass in data in the search region compared to Powheg and Sherpa. Powheg describes the data better as it is accurate to Next-to-Leading-Order in QCD as Sherpa is only accurate in leading order. Further details on this analysis can be found in [3].

\section{Vector-Boson-Scattering of WW in ATLAS}

The scattering of two massive vector bosons (VBS) is a key process to probe the nature of electroweak symmetry breaking.

Even after the discovery of the SM-like Higgs boson on 2012 many physics scenarios predict enhancements in VBS either from additional resonances or if the observed Higgs boson only 

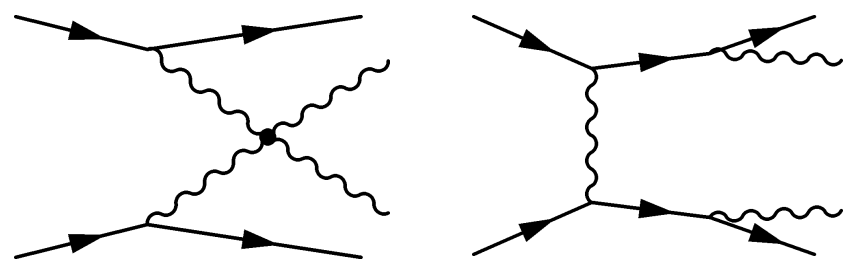

Figure 6: Left hand side: pure Vector-Boson-Scattering feynman diagram. Right hand side: pure electroweak process with a colorless exchange in the t-channel and two Vector bosons radiated from the quark lines.

partially unitarizes the $W W$ scattering amplitude.

A feynman diagram of the pure VBS process is shown on the left hand side in figure 6. This process itself is not gauge invariant and can therefore only be calculated with other pure electroweak processes, like the colorless t-channel exchange on the right hand side in figure 6. Like in the VBF process the event topology is dominated by the "tagging" jets, the high energetic forward jets, which occur directly from the incoming quarks. Also here, a high invariant mass of the dijet system is characteristic for VBS events. In addition to the two jets, two high energetic leptons from the $W$ decays and large missing transverse energy are expected. To suppress background from Drell Yan and Top-pair production the signal selection requires leptons with the same charge. The cross section for the electroweak process is measured in two different kinematic regions, which require both $m_{j j}>500 \mathrm{GeV}$. Once in the "VBS" region with an additional cut on $\Delta \eta(j j)>2.4$, where the VBS component is enhanced and in a more inclusive region without the $\Delta \eta(j j)$ cut. The measurement of the cross section is, in both regions, performed in three different channels based on the lepton flavor: $e e, \mu \mu, e \mu$. The results are shown in figure 7 , on the left hand side for the inclusive and on the right hand side for the VBS region The measured cross sections in all lepton channels separately, as well as the combination, agree well with the SM prediction.
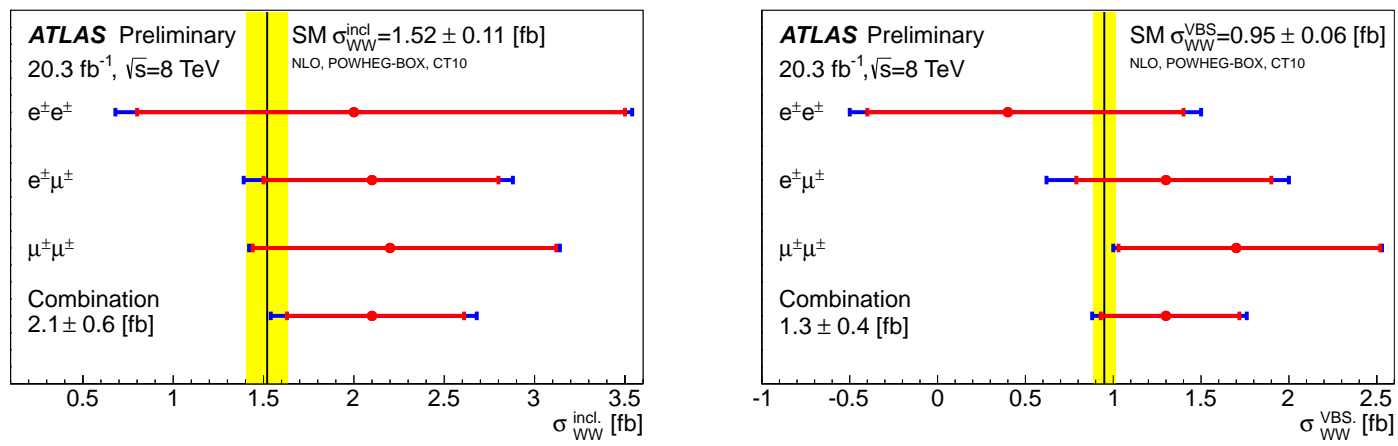

Figure 7: Results of the cross section measurements for Vector-Boson-Scattering in the inclusive (left) and in the VBS enhanced (right) phase space for the different lepton channels as well as for the combination. The inner red error band represents the statistical error, and the blue band represents the total error on each measurement. In all channels and in both phase spaces the results agree within the uncertainties with the Standard Modell.

The measured cross section in the VBS fiducial region is used to set limits on anomalous quar- 


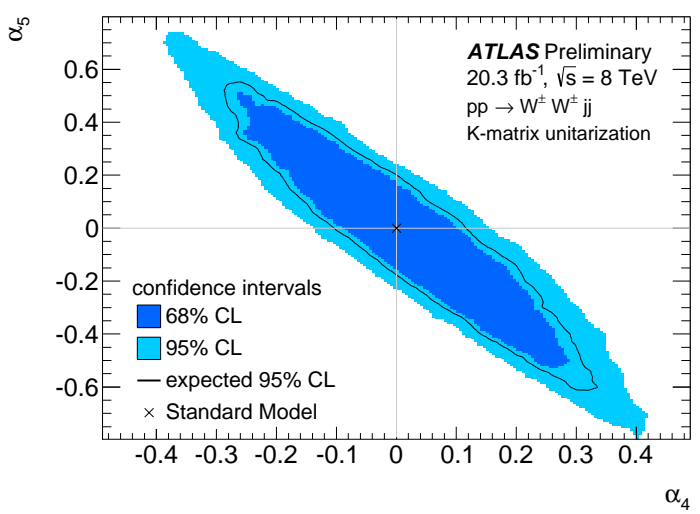

Figure 8: Limits on $\alpha_{4}$ and $\alpha_{5}$. The observed limits at $68 \%$ and 95\% CL are shown as the light and dark blue ellipses, whilst the expected limit at $95 \% \mathrm{CL}$ is given by the solid line.

tic gauge bosons coupling (aQGC) affecting vertices with four interacting $W$ bosons. The deviation from the SM can be parameterized using the parameters $\alpha_{4}$ and $\alpha_{5}$ from the chiral Lagrangian with the K-matrix unitarization scheme, a detail description can be found in [7] and [8]. Figure 8 shows the expected and observed $95 \%$ confidence intervals derived from the profile likelihood function. The limits on the parameters $\alpha_{4,5}$ are given of the one-dimensional projection at $\alpha_{5,4}=0$. The observed limits are $-0.14<\alpha_{4}<0.16$ and $-0.23<\alpha_{5}<0.24$, where the expectation is $-0.10<\alpha_{4}<0.12$ and $-0.18<\alpha_{5}<0.20$.

More details on the analysis can be found in [6].

\section{Two-photon production of WW in CMS}

This analysis performed by CMS at $\sqrt{s}=7 \mathrm{TeV}$ with an integrated luminosity of $5.05 \mathrm{fb}^{-1}$ studied the high-energy photon interaction at the LHC and is sensitive to the anomalous quartic gauge coupling at the $\gamma \gamma \rightarrow W W$ vertex. This process has a very clean signature as no extra tracks are expected at the primary vertex apart from the high energetic, charged lepton pair from the two $W$ boson decays. Furthermore large transverse missing energy is detected due to the two neutrinos from the $W$ boson decays. For further suppression of the Drell-Yan background only $e \mu$ events are selected. The efficiencies and theoretical predictions for the signal have been checked using $\gamma \gamma \rightarrow \mu \mu$ events

In the signal selection no extra tracks at the primary vertex and $p_{\mathrm{T}}\left(e^{ \pm} \mu^{\mp}\right)>30 \mathrm{GeV}$ are required. Two events pass this selection criteria while $2.2 \pm 0.4$ signal and $0.84 \pm 0.15$ background events are expected. An upper limit on the cross section at 95\%CL can be set:

$$
\sigma\left(\mathrm{pp} \rightarrow \mathrm{pW}^{+} \mathrm{W}^{-} \mathrm{p} \rightarrow \mathrm{pe}^{ \pm} \mu^{\mp} \mathrm{p}\right)<10.6 \mathrm{fb}
$$

They are consistent with the Standard Model prediction of $4.0 \pm 0.7 \mathrm{fb}$.

For the search of anomalous quartic gauge couplings, a more restricted region of $p_{\mathrm{T}}\left(e^{ \pm} \mu^{\mp}\right)>$ $100 \mathrm{GeV}$ is used. This highly suppresses the SM contribution as shown on the left hand side plot in figure 9, while it's still sensitive to anomalous quartic gauge couplings of order $10^{-4}$ for $\Lambda_{\text {cutoff }}=500 \mathrm{GeV}$. 

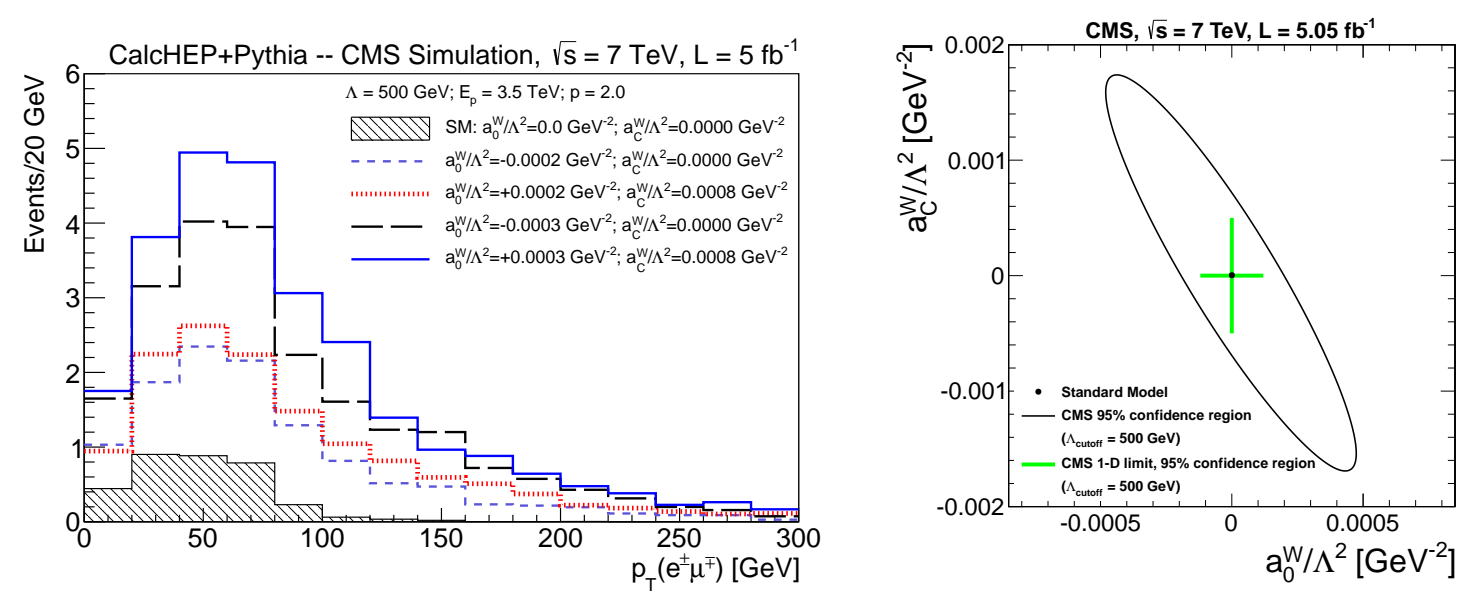

Figure 9: On the left hand side the $p_{\mathrm{T}}\left(e^{ \pm} \mu^{\mp}\right)$ distribution is shown. Especially the high $p_{\mathrm{T}}$ is sensitive to anomalous QGC. Right hand side:Excluded values of the anomalous coupling parameters $a_{0}^{\mathrm{W}} / \Lambda^{2}$ and $a_{C}^{\mathrm{W}} / \Lambda^{2}$ with $\Lambda_{\text {cutoff }}=500 \mathrm{GeV}$.

In the high- $p_{\mathrm{T}}$ tail no events are observed, which is consistent with the SM predictions of 0.14 events. One can now set a limit on each anomalous quartic gauge coupling parameter, $a_{\mathrm{C}}^{\mathrm{W}} / \Lambda^{2}$ and $a_{0}^{\mathrm{W}} / \Lambda^{2}$, with the other set to zero, further information can be found in [10]. Assuming a dipole form factor with the energy cutoff scale at $\Lambda_{\text {cutoff }}=500 \mathrm{GeV}$ the limits are:

$$
\begin{gathered}
-0.00015<a_{0}^{\mathrm{W}} / \Lambda^{2}<0.00015 \mathrm{GeV}^{-2}\left(a_{\mathrm{C}}^{\mathrm{W}} / \Lambda^{2}=0\right), \\
-0.00015<a_{\mathrm{C}}^{\mathrm{W}} / \Lambda^{2}<0.00015 \mathrm{GeV}^{-2}\left(a_{0}^{\mathrm{W}} / \Lambda^{2}=0\right)
\end{gathered}
$$

and without the form factor:

$$
\begin{gathered}
-4.0 \times 10^{-6}<a_{0}^{\mathrm{W}} / \Lambda^{2}<4.0 \times 10^{-6} \mathrm{GeV}^{-2}\left(a_{\mathrm{C}}^{\mathrm{W}} / \Lambda^{2}=0\right), \\
-1.5 \times 10^{-6}<a_{\mathrm{C}}^{\mathrm{W}} / \Lambda^{2}<1.5 \times 10^{-6} \mathrm{GeV}^{-2}\left(a_{0}^{\mathrm{W}} / \Lambda^{2}=0\right)
\end{gathered}
$$

The limits with the form factor are 20 times and the limits without the form factor even two orders of magnitude more stringent than the best limits obtained at the Tevatron. More details can be found in [9].

\section{Conclusion}

The fiducial cross section measurements of electroweak $Z+2$ jets production for proton-proton collision at $\sqrt{s}=8 \mathrm{TeV}$ using a dataset corresponding to an integrated luminosity of $20.3 \mathrm{fb}^{-1}$ collected by the ATLAS and the CMS experiment at the Large Hadron Collider have been presented. The measured cross sections are in good agreement with the Standard Model expectation. In addition, differential distributions and studies on hadronic activity have been shown. Also the cross section measurement in two fiducial regions with different sensitivity to the electroweak and the strong $W W+2$ jets production in proton-proton collision at $\sqrt{s}=8 \mathrm{TeV}$, recorded by the ATLAS experiment has been shown. Also here a good agreement with the SM prediction is found. This analysis has also been used to set the first limits on the $\alpha_{4}$ and $\alpha_{5}$ aQCG parameters. Also the search for the two photon production of $W^{+} W^{-}$using $5.05 \mathrm{fb}^{-1}$ collected by the CMS experiment 
at $\sqrt{s}=7 \mathrm{TeV}$ is shown. An upper limit on the cross section at 95\% CL and limits on the parameters $a_{\mathrm{C}}^{\mathrm{W}} / \Lambda^{2}$ and $a_{0}^{\mathrm{W}} / \Lambda^{2}$ are set.

\section{References}

[1] ATLAS Collaboration, 2008 JINST 3 S08003

[2] CMS Collaboration, 2008 JINST 3 S08004

[3] ATLAS Collaboration, JHEP04(2014)031

[4] CMS Collaboration, CMS-PAS-FSQ-12-035

[5] CMS Collaboration, JHEP10(2013)062

[6] ATLAS Collaboration, arXiv:1405.6241

[7] A. Alboteanu, W. Kilian, J. Reuter, JHEP11(2008)010

[8] T. Appelquist and C. W. Bernard, Phys. Rev. D 22 (1980) 200; A. C. Longhitano, Phys. Rev. D 22 (1980) 1166; Nucl. Phys. B188 (1981) 118.

[9] CMS Collaboration, JHEP07(2013)116

[10] G. Belanger and F. Boudjem, Phys. Lett. B 288(1992)201 